\title{
Stabilization of a Class of Continuous-Time Switched Systems with State Constraints via a Mode-Dependent Switching Method
}

\author{
Qingyu Su and Peipei Wang \\ School of Automation Engineering, Northeast Dianli University, Jilin 132012, China \\ Correspondence should be addressed to Qingyu Su; suqingyuphd@yeah.net
}

Received 13 October 2015; Accepted 18 November 2015

Academic Editor: Weiguo Xia

Copyright (C) 2015 Q. Su and P. Wang. This is an open access article distributed under the Creative Commons Attribution License, which permits unrestricted use, distribution, and reproduction in any medium, provided the original work is properly cited.

\begin{abstract}
The stability and the stabilization problems for a class of continuous-time switched systems with state constraints via a modedependent switching method are investigated. The paper presents an improved average dwell time method, which considers different decay rates of a Lyapunov function related to each of the active subsystems according to whether the saturations occur or not, respectively. It is shown that the improved average dwell time method is less conservative than the common average dwell time method. Based on the improved average dwell time method, the sufficient conditions and state feedback controllers for stabilization of the switched system are derived. A numerical example is given to illustrate the proposed approach.
\end{abstract}

\section{Introduction}

A switched system is a special class of hybrid systems that consists of a finite number of subsystems and a logical rule that orchestrates switching between these subsystems [1-3]. In the last few years, due to their success in practical applications and importance in theory development, switched systems have received much attention [4-6]. Switched systems arise in many engineering applications, such as aeronautics and astronautics systems [7, 8], mechanical systems [9], and networked control systems [10]. Owing to some safety consideration or inherent limits of devices, the majority of the practical systems have states constraints.

In the study of switched systems, one basic research topic is the issue of stability which has attracted most of the attention [11-14]. Many techniques have been studied in the study of switched systems, for example, common Lyapunov function, multiple Lyapunov functions, and switched Lyapunov functions [15-17]. However, the average dwell time method is generally recognized to have more flexibility in stabilization for switched systems [18-23].

Up to now, there exist many literatures related to stabilization of switched systems with state constraints [24-27]. However, [28] studies the stability and stabilization of switched linear systems with mode-dependent average dwell time. Reference [29] investigates the stabilization of a class of switched systems with state constraints. It is noteworthy that both of the above literatures did not discuss the stability and stabilization of switched systems with state constraints based on MDADT. In short, according to the author, the problems of stability and stabilization for continuous-time switched systems with state constraints based on mode-independent average dwell time have not been addressed in the existing literatures.

All the above observations give rise to the question of how to design the MDADT switching to stabilize the continuoustime switched systems with state constraints. This inspires us for this study.

Thus, it is necessary to investigate the stabilization problem for a class of continuous-time switched systems with state constraints based on MDADT, which is an important property for a switched system. The main contributions of this paper are given as follows: (i) an improved average dwell time method is proposed, which is less conservative than the MDADT [28] and the ADT [29]; (ii) the sufficient conditions and state feedback controllers for stabilization of continuoustime switched systems with state constraints under MDADT switching are derived.

The remainder of this paper is organized as follows. Section 2 is the problem formulation and preliminaries. Main results are given in Section 3, including sufficient condition concerning controller design and an iterative algorithm. 
Finally, a numerical example is given in Section 4. Concluding remarks are drawn in Section 5.

Notations. In this paper, the notation used is standard. $\mathbb{Z}^{+}$ denotes the set of nonnegative integers, $\mathbb{R}^{n}$ represents the $n$-dimensional space, $\|\cdot\|$ stands for the Euclidean vector norm, and $I$ is the identity matrix. Symbol $*$ denotes the symmetry elements in symmetric matrices. $\mathscr{C}^{1}$ means the space of continuously differentiable functions.

\section{Problem Formulation and Preliminaries}

Consider a class of state-constrained continuous-time switched systems given by

$$
\dot{x}(t)=h\left(A_{\sigma(t)} x(t)+B_{\sigma(t)} u_{\sigma(t)}(t)\right),
$$

where $x(t) \in \mathbb{R}^{n}$ and $u_{\sigma(t)}(t)$, respectively, denote the state vector and the control input. The two-matrix pair $\left(A_{p}, B_{p}\right)$, $\forall \sigma(t)=p \in \mathscr{L}$, represents the $p$ th subsystem or $p$ th mode of (1); symbol $h(\cdot)$ denotes the saturation function. $\sigma(t)$ is a piecewise constant function of time, which takes its values in the finite set $\mathscr{L}=\{1,2, \ldots, N\} ; N>1$ is the number of subsystems. In addition, for a switching sequence $0<t_{1}<$ $\cdots<t_{i-1}<t_{i}<\cdots$, symbol $t_{i}$ means the moment of the $i$ th switching. When $t \in\left(t_{i-1}, t_{i}\right)$, we say the $\sigma\left(t_{i-1}\right)$ th subsystem is active.

Generally speaking, the ideal state feedback is treated as $u_{\sigma(t)}(t)=F_{\sigma(t)} x(t)$, where $F_{\sigma(t)}$ is the controller gain to be determined. Then, the resulting closed-loop system is described by

$$
\dot{x}(t)= \begin{cases}\mathscr{A}_{p} x(t), & \text { non-constrained } \\ \overline{\mathscr{A}}_{p} x(t), & \text { constrained }\end{cases}
$$

where $\mathscr{A}_{p}:=A_{p}+B_{p} F_{p}$, which represents the closed-loop system matrix of the $p$ th subsystem.

About the saturation function $h(\cdot)$, we transform it into the vertex of a convex hull to handle the saturations [30].

Symbol $U_{n}$ denotes the set of $n \times n$ diagonal matrices. The diagonal elements of $U_{n}$ are 0 or 1 . Assuming that every element of $U_{n}$ is marked as $U_{s}, U_{s}^{-}=I-U_{s}, s \in\{1,2, \ldots$, $\left.2^{n-1}, 2^{n}\right\}$. Therefore, we have

$$
h(A x) \in \operatorname{co}\left\{U_{s}(A x)+U_{s}^{-} L\right\},
$$

and the diagonal elements of row diagonally dominant matrix $L$ are negative.

Now, let us first revisit the following definition and lemmas for later development.

Definition 1 (see [19]). For a switching signal $\sigma(t)$ and each $t_{2} \geq t_{1} \geq 0$, let $N_{\sigma(t)}\left(t_{2}, t_{1}\right)$ denote the number of discontinuities of $\sigma(t)$ in the open interval $\left(t_{1}, t_{2}\right)$. We say that $\sigma(t)$ has an average dwell time $\tau_{a}$ if there exist two positive numbers $N_{0}$ and $\tau_{a}$ such that

$$
N_{\sigma(t)}\left(t_{2}, t_{1}\right) \leq N_{0}+\frac{t_{2}-t_{1}}{\tau_{a}}, \quad \forall t_{2} \geq t_{1} \geq 0 .
$$

Remark 2. Definition 1 means that if there exists a positive number $\tau_{a}$ such that a switching signal has the ADT property, the ADT between any two consecutive switching signals is no smaller than a common constant $\tau_{a}$ for all subsystems.

Lemma 3 (see [29]). Consider a continuous-time state-constrained switched system:

$$
\dot{x}(t)=f_{\sigma(t)}(x(t)), \quad x(t) \in \Omega \subset \mathbb{R}^{n}, \sigma(t)=p \in \mathscr{P},
$$

with $f_{p}(0)=0, \forall p \in \mathscr{P}$. Assume that all trajectories remain inside $\Omega$. Let $\alpha, \beta$, and $\mu$ be some given constants satisfying $\alpha>\beta>0, \mu \geq 1$. If there exist some piecewise continuous functions $V_{p}(x(t)): \Omega \rightarrow \mathbb{R}$, for all $p \in \mathscr{P}$, such that

$$
\begin{gathered}
\varphi_{1}(\|x(t)\|) \leq V_{p}(x(t)) \leq \varphi_{2}(\|x(t)\|), \quad \forall x(t) \in \Omega, \\
\dot{V}_{p}(x(t)) \leq \begin{cases}-\alpha V_{p}(x(t)), & \forall t \in \mathscr{T}_{f}\left(t_{i}, t_{i+1}\right), \\
-\beta V_{p}(x(t)), & \forall t \in \mathscr{T}_{s}\left(t_{i}, t_{i+1}\right),\end{cases}
\end{gathered}
$$

and $\forall\left(\sigma\left(t_{i}\right)=p, \sigma\left(t_{i}^{-}\right)=q\right) \in \mathscr{P} \times \mathscr{P}, p \neq q$,

$$
V_{p}\left(x\left(t_{i}\right)\right) \leq \mu V_{q}\left(x\left(t_{i}\right)\right), \quad \forall x(t) \in \Omega,
$$

where $\varphi_{1}$ and $\varphi_{2}$ are some class $\mathscr{K}$ functions, then the stateconstrained switched system (5) is GUAS for any switching signal with ADT

$$
\begin{array}{r}
\tau_{a} \geq \tau_{a}^{*}=\frac{\ln \mu}{\zeta}, \\
\frac{\mathscr{T}_{f}\left(t_{0}, t\right)}{\mathscr{T}_{s}\left(t_{0}, t\right)} \geq \frac{\zeta-\beta}{\alpha-\zeta}>0 .
\end{array}
$$

Lemma 4 (see [28]). Consider the continuous-time switched system $\dot{x}(t)=f_{\sigma(t)}(x(t)), \sigma(t) \in \mathscr{P}$, and let $\lambda_{p}>0$ and $\mu_{p}>1$ be given constants. Supposing that there exist positive definite $\mathscr{C}^{1}$ functions $V_{\sigma(t)}: \mathbb{R}^{n} \rightarrow \mathbb{R}$ and two class $\mathscr{K}_{\infty}$ functions $\kappa_{1 p}$ and $\kappa_{2 p}, p \in \mathcal{S}$,

$$
\begin{gathered}
\kappa_{1 p}(\|x(t)\|) \leq V_{p}(x(t)) \leq \kappa_{2 p}(\|x(t)\|), \\
\dot{V}_{p}(x(t)) \leq-\lambda_{p} V_{p}(x(t)),
\end{gathered}
$$

and $\forall\left(\sigma\left(t_{i}\right)=p, \sigma\left(t_{i}^{-}\right)=q\right) \in \mathcal{S} \times \mathcal{S}, p \neq q$,

$$
V_{p}\left(x\left(t_{i}\right)\right) \leq \mu_{p} V_{q}\left(x\left(t_{i}\right)\right),
$$

then the system is GUAS for any switching signal with MDADT:

$$
\tau_{a p} \geq \tau_{a p}^{*}=\frac{\ln \mu_{p}}{\lambda_{p}} .
$$

\section{Main Results}

In this section, we first establish the stability conclusion for the continuous-time switched systems with state constraints based on MDADT in Lemma 7. The following definition is important for our result. 
Definition 5 (see [28]). For a switching signal $\sigma(t)$ and each $T \geq 0$, let $N_{\sigma p}(T, 0)$ denote the switching numbers that the $p$ th subsystem is activated over the interval $[0, T]$ and let $T_{p}(T, 0)$ denote the total running time of the $p$ th subsystem over the interval $[0, T], p \in \mathcal{S}$. We say that $\sigma(t)$ has a mode-dependent average dwell time $\tau_{a p}$ if there exist two positive numbers $N_{0 p}$ (we call $N_{0 p}$ the mode-dependent chatter bounds here) and $\tau_{a p}$ such that

$$
N_{\sigma p}(T, 0) \leq N_{0 p}+\frac{T_{p}(T, 0)}{\tau_{a p}}, \quad \forall T \geq 0 .
$$

Remark 6. Definition 5 constructs a novel set of switching signals with a MDADT property: it means that if there exist positive numbers $\tau_{a p}, p \in \mathcal{S}$, such that a switching signal has the MDADT property, we only require that the average time among the intervals associated with the $p$ th subsystem is larger than $\tau_{a p}$ (note that the intervals here are not adjacent), where $N_{\sigma}(T, 0)=\sum_{p=1}^{M} N_{\sigma p}(T, 0)$.

Next, Lemma 7 presents the stability result for the continuous-time state-constrained switched systems under MDADT switching.

Lemma 7. Consider the following switched systems:

$$
\dot{x}(t)=f_{\sigma(t)}(x(t)),
$$

where $x(t) \in \Phi \subset \mathbb{R}^{n}$, and let $\lambda_{p}>\gamma_{p}>0$ and $\mu_{p} \geq 1, p \in$ $\mathscr{L}$, be given constants. Supposing that there exist $\mathscr{C}^{1}$ function
$V_{\sigma(t)}(x(t)): \Phi \rightarrow \mathbb{R}$ and some class $\mathscr{W}$ functions $w_{1 p}$ and $w_{2 p}, \forall p \in \mathscr{L}$, then

$$
\begin{aligned}
w_{1 p}(\|x(t)\|) & \leq V_{p}(x(t)) \leq w_{2 p}(\|x(t)\|), \\
\dot{V}_{p}(x(t)) & \leq-\lambda_{p} V_{p}(x(t)), \quad \forall t \in R_{p}\left(t_{i-1}, t_{i}\right) ; \\
\dot{V}_{p}(x(t)) & \leq-\gamma_{p} V_{p}(x(t)), \quad \forall t \in S_{p}\left(t_{i-1}, t_{i}\right),
\end{aligned}
$$

and $\forall\left(\sigma\left(t_{i}\right)=p, \sigma\left(t_{i}^{-}\right)=q\right) \in \mathscr{L} \times \mathscr{L}, p \neq q, i \in \mathbb{Z}^{+}$,

$$
V_{p}\left(x\left(t_{i}\right)\right) \leq \mu_{p} V_{q}\left(x\left(t_{i}\right)\right)
$$

then the switched system (14) is GUAS under any MDADT switching signal

$$
\tau_{a p} \geq \tau_{a p}^{*}=\frac{\ln \mu_{p}}{\rho},
$$

where $\lambda_{p} R_{p}\left(t_{0}, t\right)+\gamma_{p} S_{p}\left(t_{0}, t\right) \geq \rho T_{p}$.

Proof. From the above, we know that the system states can be divided into the saturated state and the nonsaturated state. For example, when $t>t_{s} \in S_{p}\left(t_{i-1}, t_{i}\right)$, that is to say, the system state is saturated, then we can conclude from that that

$$
V_{p}(x(t))-e^{-\gamma_{p}\left(t-t_{s}\right)} V_{p}\left(x\left(t_{s}\right)\right)<0 .
$$

The inequality for the nonsaturated state can be obtained using similar techniques. Therefore, when $t>t_{i}$, together with (17) and (19), it implies

$$
\begin{aligned}
V_{\sigma\left(t_{i}\right)}(x(t)) & \leq e^{-\lambda_{\sigma\left(t_{i}\right)} R_{\sigma\left(t_{i}\right)}\left(t_{i}, t\right)} e^{-\gamma_{\sigma\left(t_{i}\right)} S_{\sigma\left(t_{i}\right)}\left(t_{i}, t\right)} V_{\sigma\left(t_{i}\right)}\left(x\left(t_{i}\right)\right) \leq \mu_{\sigma\left(t_{i}\right)} e^{-\lambda_{\sigma\left(t_{i}\right)} R_{\sigma\left(t_{i}\right)}\left(t_{i}, t\right)} e^{\left.-\gamma_{\sigma\left(t_{i}\right)}\right)_{\sigma\left(t_{i}\right)}\left(t_{i}, t\right)} V_{\sigma\left(t_{i-1}\right)}\left(x\left(t_{i}^{-}\right)\right) \\
& \leq \mu_{\sigma\left(t_{i}\right)} e^{-\lambda_{\sigma\left(t_{i}\right)} R_{\sigma\left(t_{i}\right)}\left(t_{i}, t\right)-\lambda_{\sigma\left(t_{i-1}\right)} R_{\sigma\left(t_{i-1}\right)}\left(t_{i-1}, t_{i}\right)} e^{-\gamma_{\sigma\left(t_{i}\right)} S_{\sigma\left(t_{i}\right)}\left(t_{i}, t\right)-\gamma_{\sigma\left(t_{i-1}\right)} S_{\sigma\left(t_{i-1}\right)}\left(t_{i-1}, t_{i}\right)} V_{\sigma\left(t_{i-1}\right)}\left(x\left(t_{i-1}\right)\right) \\
& \leq \mu_{\sigma\left(t_{i}\right)} \mu_{\sigma\left(t_{i-1}\right)} e^{-\lambda_{\sigma\left(t_{i}\right)} R_{\sigma\left(t_{i}\right)}\left(t_{i}, t\right)-\lambda_{\sigma\left(t_{i-1}\right)} R_{\sigma\left(t_{i-1}\right)}\left(t_{i-1}, t_{i}\right)} e^{-\gamma_{\sigma\left(t_{i}\right)} S_{\sigma\left(t_{i}\right)}\left(t_{i}, t\right)-\gamma_{\sigma\left(t_{i-1}\right)} S_{\sigma\left(t_{i-1}\right)}\left(t_{i-1}, t_{i}\right)} V_{\sigma\left(t_{i-2}\right)}\left(x\left(t_{i-1}^{-}\right)\right) \leq \cdots \\
& \leq \prod_{p=1}^{M} \mu_{p}^{N_{\sigma p}} e^{-\sum_{p=1}^{M}\left[\lambda_{p} R_{p}\left(t_{0}, t\right)+\gamma_{p} S_{p}\left(t_{0}, t\right)\right]} V_{\sigma\left(t_{0}\right)}\left(x\left(t_{0}\right)\right) .
\end{aligned}
$$

In summary, this implies

$$
\begin{aligned}
V_{\sigma\left(t_{i}\right)}(x(t)) & \leq \prod_{p=1}^{M} \mu_{p}^{N_{\sigma p}} e^{-\rho T_{p}} V_{\sigma\left(t_{0}\right)}\left(x\left(t_{0}\right)\right) \\
& \leq \prod_{p=1}^{M} \mu_{p}^{N_{0 p}} \mu_{p}^{T_{p} / \tau_{a p}} e^{-\rho T_{p}} V_{\sigma\left(t_{0}\right)}\left(x\left(t_{0}\right)\right) \\
& \leq \prod_{p=1}^{M} \mu_{p}^{N_{0 p}}\left(\mu_{p}^{1 / \tau_{a p}} e^{-\rho}\right)^{T_{p}} V_{\sigma\left(t_{0}\right)}\left(x\left(t_{0}\right)\right) .
\end{aligned}
$$

Therefore, supposing that the switching signal satisfies

$$
\tau_{a p} \geq \tau_{a p}^{*}=\frac{\ln \mu_{p}}{\rho},
$$

we have $\mu_{p}^{1 / \tau_{a p}} e^{-\rho} \leq \mu_{p}^{\rho / \ln \mu_{p}} e^{-\rho}=1$. With $V_{\sigma\left(t_{i}\right)}(x(t))$ convergence to zero along with $t \rightarrow \infty$, the asymptotic stability also can be derived.

Remark 8. In Lemma 7, assume that $\lambda_{p}>\gamma_{p}$, and symbols $\lambda_{p}$ and $\gamma_{p}$ respectively denote the different decay rates of a Lyapunov function related to each of the active subsystems according to whether the saturations occur or not. For convenience of understanding, $R_{p}\left(t_{i-1}, t_{i}\right)$ and $S_{p}\left(t_{i-1}, t_{i}\right)$ represent separately the total time of the $p$ th subsystem during which the state is saturated or nonsaturated within $\left[t_{i-1}, t_{i}\right)$. In addition, we define $R_{p}\left(t_{0}, t\right)=\sum_{f=1}^{i} R_{p}\left(t_{f-1}, t_{f}\right)+R_{p}\left(t_{i}, t\right)$ and $S_{p}\left(t_{0}, t\right)=\sum_{f=1}^{i} S_{p}\left(t_{f-1}, t_{f}\right)+S_{p}\left(t_{i}, t\right)$. 
Remark 9. It can be seen from Lemma 3 that the parameters $\alpha, \beta$, and $\mu$ are the same for all subsystems according to whether the saturations occur or not. However, the parameters $\lambda_{p}, \gamma_{p}$, and $\mu_{p}$ prescribed in Lemma 7 are modedependent; in other words, symbol $\lambda_{p}$ and $\gamma_{p}$, respectively, denote the different decay rates of a Lyapunov function related to each of the active subsystems according to whether the saturations occur or not. Therefore, we can conclude that $\tau_{a p}^{*} \leq \tau_{a}^{*}$, from (7)-(9) and (16)-(18), and the modedependent features would reduce the conservativeness existing in Lemma 3.

Remark 10. It is worth nothing that the improved ADT (18) is always smaller than ADT (9) and (12). What is more, if we choose $\lambda_{p}=\gamma_{p}$ and $\lambda_{p}=\alpha, \gamma_{p}=\beta$, and $\mu_{p}=\mu$ then (18) will reduce to (12) and (9), respectively. Therefore, Lemmas 3 and 4 can be regarded as two special cases of Lemma 7. It is clear that Lemma 7 presents a more general stability criterion than Lemmas 3 and 4 which corresponds to the special case of $\lambda_{p}=\gamma_{p}$ and $\lambda_{p}=\alpha, \gamma_{p}=\beta, \mu_{p}=\mu$, and $\forall p \in \mathscr{L}$.

In total, we can infer from Remarks 9 and 10 that the MDADT switching signal has great flexibility superiorities for a switched system with state constraints.

Now, based on the consequences obtained above, we present the stability condition for continuous-time switched systems with state constraints based on MDADT.

Theorem 11. Consider the following switched system:

$$
\dot{x}(t)=h\left(A_{\sigma(t)} x(t)\right)
$$

where $\sigma(t)=p \in \mathscr{L}$, and let $\lambda_{p}>\gamma_{p}>0$ and $\mu_{p} \geq 1, p \in \mathscr{L}$, be given constants. $\forall(p, q) \in \mathscr{L} \times \mathscr{L}, p \neq q$ and $U_{s} \neq I$. Supposing that there exist matrices $Q_{p}>0$ and row diagonally dominant matrices $L_{p}$, where $\left|l_{i i}\right|>\sum_{j=1, j \neq i}^{n}\left|l_{i j}\right|$ and $l_{i i}<0$, $\forall i \in \mathcal{N}$,

$$
\begin{aligned}
& A_{p}^{T} Q_{p}+Q_{p} A_{p}+\lambda_{p} Q_{p}<0, \\
& \left(U_{s} A_{p}+U_{p}^{-} L_{p}\right)^{T} Q_{p}+Q_{p}\left(U_{s} A_{p}+U_{s}^{-} L_{p}\right)+\gamma_{p} Q_{p} \\
& \quad<0 \\
& Q_{p}-\mu_{p} Q_{q}<0
\end{aligned}
$$

therefore, the continuous-time switched system with state constraints (23) is GUAS with MDADT satisfying (18).

Proof. Here, consider the following Lyapunov function:

$$
V_{p}(x(t))=x^{T}(t) Q_{p} x(t)
$$

where $\forall \sigma(t)=p \in \mathscr{L}$. From Lemma 7, we know that the system states can be divided into the saturated state and the nonsaturated state. Therefore, when $t \in R_{p}\left(t_{i-1}, t_{i}\right)$, in other words, the system state is nonsaturated, we can conclude from that that

$$
\begin{aligned}
& \dot{V}_{p}(x(t))+\lambda_{p} V_{p}(t) \\
& \quad=x^{T}(t)\left(\lambda_{p} Q_{p}+A_{p}^{T} Q_{p}+Q_{p} A_{p}\right) x(t)<0, \\
& V_{p}\left(x\left(t_{i}\right)\right)-\mu_{p} V_{q}\left(x\left(t_{i}\right)\right) \\
& \quad=x^{T}\left(t_{i}\right)\left(Q_{p}-\mu_{p} Q_{q}\right) x\left(t_{i}\right)<0 .
\end{aligned}
$$

The inequality for the saturated state can be obtained using similar techniques:

$$
\begin{aligned}
& \dot{V}_{p}(x(t))+\gamma_{p} V_{p}(t) \\
& \quad=x^{T}(t)\left(\gamma_{p} Q_{p}+\widetilde{A}_{p}^{T} Q_{p}+Q_{p} \widetilde{A}_{p}\right) x(t)<0,
\end{aligned}
$$

where $\widetilde{A}_{p}=U_{s} A_{p}+U_{s}^{-} L_{p}$. By simplifying the above three inequalities, we can get (24). Therefore, the continuous-time switched system with state constraints (23) is GUAS with MDADT satisfying (18).

Next, we introduce the condition of stabilizing controller for continuous-time switched systems with state constraints based on MDADT.

Theorem 12. Consider the same switched system (1), where $\sigma(t)=p \in \mathscr{L}$, and let $\lambda_{p}>\gamma_{p}>0$ and $\mu_{p} \geq 1, p \in \mathscr{L}$, be given constants. $\forall(p, q) \in \mathscr{L} \times \mathscr{L}, p \neq q$ and $U_{s} \neq I$. Suppose that there exist matrices $P_{p}>0$ and $P_{q}>0$ and row diagonally dominant matrices $L_{p}$ and $T_{p}$, where $\left|l_{i i}\right|>\sum_{j=1, j \neq i}^{n}\left|l_{i j}\right|$ and $l_{i i}<0, \forall i \in \mathcal{N}$,

$$
\begin{aligned}
& P_{p} A_{p}^{T}+T_{p}^{T} B_{p}^{T}+A_{p} P_{p}+B_{p} T_{p}+\lambda_{p} P_{p}<0, \\
& P_{p} A_{p}^{T} U_{s}^{T}+T_{p}^{T} B_{p} U_{s}^{T}+U_{p} L_{p} U_{s}^{-T}+U_{s} A_{p} P_{p} \\
& \quad+U_{s} B_{p} T_{p}+U_{s}^{-} L_{p} P_{p}+\gamma_{p} P_{p}<0, \\
& P_{p}-\mu_{p} P_{q}<0 ;
\end{aligned}
$$

then, the continuous-time switched system with state constraints (1) is GUAS with MDADT satisfying (18). Therefore, if (28), (29), and (30) have a solution, the controller gains can be obtained by

$$
F_{p}=T_{p} P_{p}^{-1}
$$

Proof. From the closed-loop system (2) and Theorem 11, we have

$$
\begin{aligned}
& \left(A_{p}+B_{p} F_{p}\right)^{T} Q_{p}+Q_{p}\left(A_{p}+B_{p} F_{p}\right)<-\lambda_{p} Q_{p} \\
& {\left[U_{s}\left(A_{p}+B_{p} F_{p}\right)+U_{s}^{-} L_{p}\right]^{T} Q_{p}} \\
& \quad+Q_{p}\left[U_{s}\left(A_{p}+B_{p} F_{p}\right)+U_{s}^{-} L_{p}\right]<-\gamma_{p} Q_{p}, \\
& Q_{p}<\mu_{p} Q_{q} .
\end{aligned}
$$


For simplifying the above inequalities, we can pre- and postmultiply $Q_{p}^{-1}$ to (32) and (33). Next, we define $T_{p}=F_{p} Q_{p}^{-1}$ and $P_{p}=Q_{p}^{-1}$, such that (28), (29), and (30) are proved to be established. Therefore, if (28), (29), and (30) have a solution, the controller gains can be obtained by $F_{p}=T_{p} P_{p}^{-1}$. Then, the continuous-time switched system with state constraints (1) is GUAS with MDADT satisfying (18).

Remark 13. Theorem 12 is a prolongation of [29, Theorem 3], which deals with the stabilization problem of continuoustime switched systems with state constraints. From Remarks 9 and 10 , using $\lambda_{p}, \gamma_{p}, \mu_{p}$ under MDADT switching to design feasible controllers has more flexibility and less conservativeness.

Finally, we give an iterative LMI algorithm for verifying the sufficient conditions of theorems for the continuous-time case. Here, we briefly describe the iterative LMI algorithm: Step 1, select a $E_{p}>0$ and solve $P_{p}$ and $T_{p}$ from the Lyapunov equation (28); Step 2, using $P_{p}$ and $T_{p}$ obtained previously, solve the LMI optimization problem (29) for $L_{p}$ and $\xi$; Step 3, using $L_{p}$ obtained in the previous step, solve the LMI optimization problems (28) and (29) for $P_{p}$ and $T_{p}$ and $\xi$; Step 4, if $\xi \leq 0$, system (2) is GUAS at the origin. And the current $F_{p}$ is the calculated feedback again. Otherwise, no result can be obtained. A different $E_{p}$ may be selected and the algorithm may be repeated from Step 1. For more details on the iterative LMI algorithm, please refer to the reference literature [30].

\section{Numerical Example}

In this section, a numerical example of the continuous-time switched system with state constraints based on MDADT is presented to show the effectiveness of the developed approaches.

Example. Consider the following switched systems:

$$
\dot{x}(t)=h\left(A_{\sigma(t)} x(t)\right),
$$

where $\sigma(t) \in \mathscr{L}=\{1,2\}$ and

$$
\begin{aligned}
& A_{1}=\left[\begin{array}{ccc}
-2.0 & 0.1 & -0.5 \\
-0.4 & -0.9 & 0.7 \\
0.3 & -1.0 & -0.4
\end{array}\right], \\
& A_{2}=\left[\begin{array}{ccc}
-0.7 & -1.5 & 0.5 \\
2.0 & -1.0 & 1.2 \\
1.8 & 1.0 & -1.8
\end{array}\right] .
\end{aligned}
$$

We set the parameters $\lambda_{1}=0.3, \gamma_{1}=0.1$, and $\mu_{1}=1.4 ; \lambda_{2}=$ $0.2, \gamma_{2}=0.14$, and $\mu_{2}=1.5 ; \rho=0.15$ and $R_{1}(0, t) / S_{1}(0, t)=$ $0.4 ; R_{2}(0, t) / S_{2}(0, t)=0.2$. In Theorem 11,

$$
Q_{1}=\left[\begin{array}{ccc}
32.1545 & -3.8173 & 8.9418 \\
-3.8173 & 22.8047 & -4.0990 \\
8.9418 & -4.0990 & 22.1684
\end{array}\right],
$$

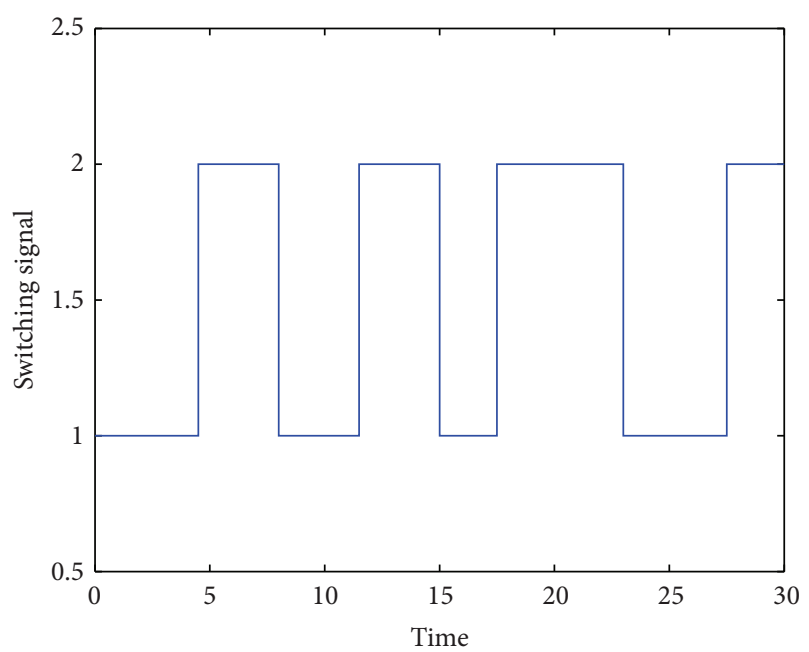

FIGURE 1: $\tau_{a 1}=2.5$ and $\tau_{a 2}=2.8$.

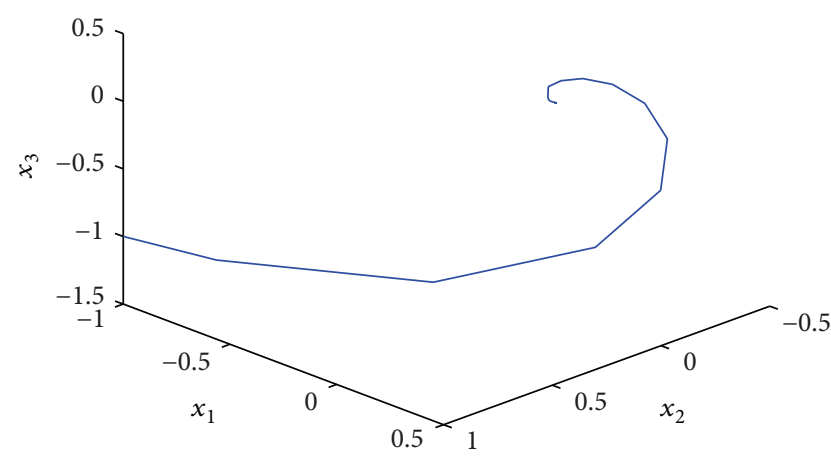

Figure 2: Saturated trajectory of system (35).

$$
\begin{aligned}
& Q_{2}=\left[\begin{array}{ccc}
41.1823 & -4.5734 & 12.2986 \\
-4.5734 & 21.8687 & -4.0101 \\
12.2986 & -4.0101 & 21.1007
\end{array}\right], \\
& L_{1}=\left[\begin{array}{ccc}
-3.8256 & 0.4542 & -1.0639 \\
0.6506 & -3.8869 & -0.6986 \\
-1.4754 & 0.6764 & -3.6579
\end{array}\right], \\
& L_{2}=\left[\begin{array}{ccc}
-3.8032 & -0.4224 & -1.1358 \\
0.8064 & -3.8559 & 0.7071 \\
-1.9884 & 0.6484 & -3.4116
\end{array}\right] .
\end{aligned}
$$

When the MDADT switching signal satisfies $\tau_{a 1} \geq 2.4549$ and $\tau_{a 2} \geq 2.6273$, the switched system with state constraints (35) is GUAS. So, we give $\tau_{a 1}=2.5$ and $\tau_{a 2}=2.8$ in Figure 1 . Figure 2 shows that despite $x(t)$ has occurred saturations, with the initial state $x_{0}=[-1,1,-1]^{T}$ and the MDADT switching signal, the continuous-time switched system with state constraints is GUAS. From the example, we demonstrate the effectiveness of our proposed method. 


\section{Conclusion}

The stability and the stabilization problems for a class of continuous-time switched systems with state constraints via a mode-dependent switching method have been investigated in continuous-time context. An improved average dwell time method is proposed. It is shown that the improved average dwell time method is less conservative than the common average dwell time method. Then, based on the improved average dwell time method, the sufficient conditions and state feedback controllers for stabilization of the switched system are derived. Finally, a numerical example is given to illustrate the proposed approach.

\section{Conflict of Interests}

The authors declare that there is no conflict of interests regarding the publication of this paper.

\section{Acknowledgments}

This work is supported by the Funds of National Science of China (Grant nos. 61403075 and 61503071) and the Natural Science Foundation of Jilin Province (Grant no. 20140520060JH).

\section{References}

[1] D. Liberzon, Switching in Systems and Control, Birkhäuser, Boston, Mass, USA, 2003.

[2] D. Liberzon and A. S. Morse, "Basic problems in stability and design of switched systems," IEEE Control Systems Magazine, vol. 19, no. 5, pp. 59-70, 1999.

[3] Z. D. Sun and S. S. Ge, "Analysis and synthesis of switched linear control systems," Automatica, vol. 41, no. 2, pp. 181-195, 2005.

[4] L. X. Zhang and H. J. Gao, "Asynchronously switched control of switched linear systems with average dwell time," Automatica, vol. 46, no. 5, pp. 953-958, 2010.

[5] Q. Y. Su, M. S. Wang, G. M. Dimirovski, X. X. Dong, and J. Zhao, "Tracking of output-constrained switched nonlinear systems in strict-feedback form," in Proceedings of the 31st Chinese Control Conference (CCC '12), pp. 367-372, IEEE, Hefei, China, July 2012.

[6] Y. Y. Liu and J. Zhao, "Stabilization of switched nonlinear systems with passive and non-passive subsystems," Nonlinear Dynamics, vol. 67, no. 3, pp. 1709-1716, 2012.

[7] H. Lin and P. J. Antsaklis, "Stability and stabilizability of switched linear systems: a survey of recent results," IEEE Transactions on Automatic Control, vol. 54, no. 2, pp. 308-322, 2009.

[8] X. Zhao, L. Zhang, P. Shi, and M. Liu, "Stability of switched positive linear systems with average dwell time switching," Automatica, vol. 48, no. 6, pp. 1132-1137, 2012.

[9] M. B. Yazdi and M. R. Jahed-Motlagh, "Stabilization of a CSTR with two arbitrarily switching modes using modal state feedback linearization," Chemical Engineering Journal, vol. 155, no. 3, pp. 838-843, 2009.

[10] H. Lin and P. J. Antsaklis, "Stability and persistent disturbance attenuation properties for a class of networked control systems: switched system approach," International Journal of Control, vol. 78, no. 18, pp. 1447-1458, 2005.
[11] M. S. Branicky, "Multiple Lyapunov functions and other analysis tools for switched and hybrid systems," IEEE Transactions on Automatic Control, vol. 43, no. 4, pp. 475-482, 1998.

[12] K. S. Narendra and J. Balakrishnan, "A common Lyapunov function for stable LTI systems with commuting A-matrices," IEEE Transactions on Automatic Control, vol. 39, no. 12, pp. 2469-2471, 1994.

[13] L. J. Long and J. Zhao, "Global stabilisation of switched nonlinear systems in $p$-normal form with mixed odd and even powers," International Journal of Control, vol. 84, no. 10, pp. 1612-1626, 2011.

[14] L. Fainshil, M. Margaliot, and P. Chigansky, "On the stability of positive linear switched systems under arbitrary switching laws," IEEE Transactions on Automatic Control, vol. 54, no. 4, pp. 897-899, 2009.

[15] J. Zhao and D. J. Hill, "On stability, $L_{2}$-gain and $H_{\infty}$ control for switched systems," Automatica, vol. 44, no. 5, pp. 1220-1232, 2008.

[16] J. Daafouz, P. Riedinger, and C. Iung, "Stability analysis and control synthesis for switched systems: a switched Lyapunov function approach," IEEE Transactions on Automatic Control, vol. 47, no. 11, pp. 1883-1887, 2002.

[17] X. Zhao, P. Shi, and L. Zhang, "Asynchronously switched control of a class of slowly switched linear systems," Systems \& Control Letters, vol. 61, no. 12, pp. 1151-1156, 2012.

[18] J. Lian and M. Wang, "Sliding-mode control of switched delay systems with nonlinear perturbations: average dwell time approach," Nonlinear Dynamics, vol. 62, no. 4, pp. 791-798, 2010.

[19] J. P. Hespanha and A. S. Morse, "Stability of switched systems with average dwell time," in Proceedings of the 38th IEEE Conference on Decision and Control, pp. 2655-2660, Phoenix, Ariz, USA, 1999.

[20] G. S. Zhai, B. Hu, K. Yasuda, and A. N. Michel, "Stability analysis of switched systems with stable and unstable subsystems: an average dwell time approach," International Journal of Systems Science, vol. 32, no. 8, pp. 1055-1061, 2001.

[21] X.-M. Sun, J. Zhao, and D. J. Hill, "Stability and $L_{2}$-gain analysis for switched delay systems: a delay-dependent method," Automatica, vol. 42, no. 10, pp. 1769-1774, 2006.

[22] Q. Y. Su, L. J. Long, and J. Zhao, "Stabilization of state-constrained switched nonlinear systems in p-normal form," International Journal of Robust and Nonlinear Control, vol. 24, no. 10, pp. 1550-1562, 2014.

[23] Q. Su and J. Zhao, " $H_{\infty}$ control for a class of discrete-time switched systems with state constraints," Proceedings of the Institution of Mechanical Engineers Part I: Journal of Systems and Control Engineering, vol. 226, no. 11, pp. 1301-1310, 2012.

[24] J. Zhao and G. M. Dimirovski, "Quadratic stability of a class of switched nonlinear systems," IEEE Transactions on Automatic Control, vol. 49, no. 4, pp. 574-578, 2004.

[25] K. P. Tee, S. S. Ge, and F. E. H. Tay, "Adaptive control of electrostatic microactuators with bidirectional drive," IEEE Transactions on Control Systems Technology, vol. 17, no. 2, pp. 340-352, 2009.

[26] H. Richter, "A multi-regulator sliding mode control strategy for output-constrained systems," Automatica, vol. 47, no. 10, pp. 2251-2259, 2011.

[27] A. S. Morse, "Supervisory control of families of linear setpoint controllers Part I. Exact matching," IEEE Transactions on Automatic Control, vol. 41, no. 10, pp. 1413-1431, 1996. 
[28] X. Zhao, L. Zhang, P. Shi, and M. Liu, "Stability and stabilization of switched linear systems with mode-dependent average dwell time," IEEE Transactions on Automatic Control, vol. 57, no. 7, pp. 1809-1815, 2011.

[29] Q. Su and J. Zhao, "Stabilization of a class of switched systems with state constraints," Nonlinear Dynamics, vol. 70, no. 2, pp. 1499-1510, 2012.

[30] L. Hou and A. N. Michel, "Asymptotic stability of systems with saturation constraints," IEEE Transactions on Automatic Control, vol. 43, no. 8, pp. 1148-1154, 1998. 


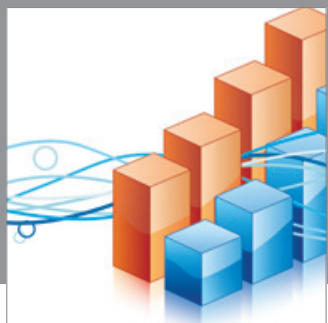

Advances in

Operations Research

mansans

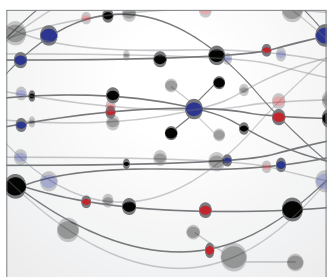

The Scientific World Journal
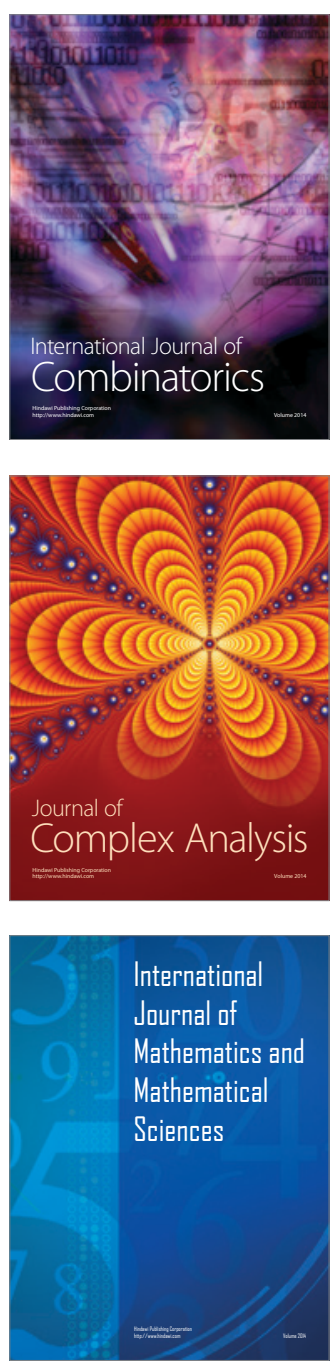
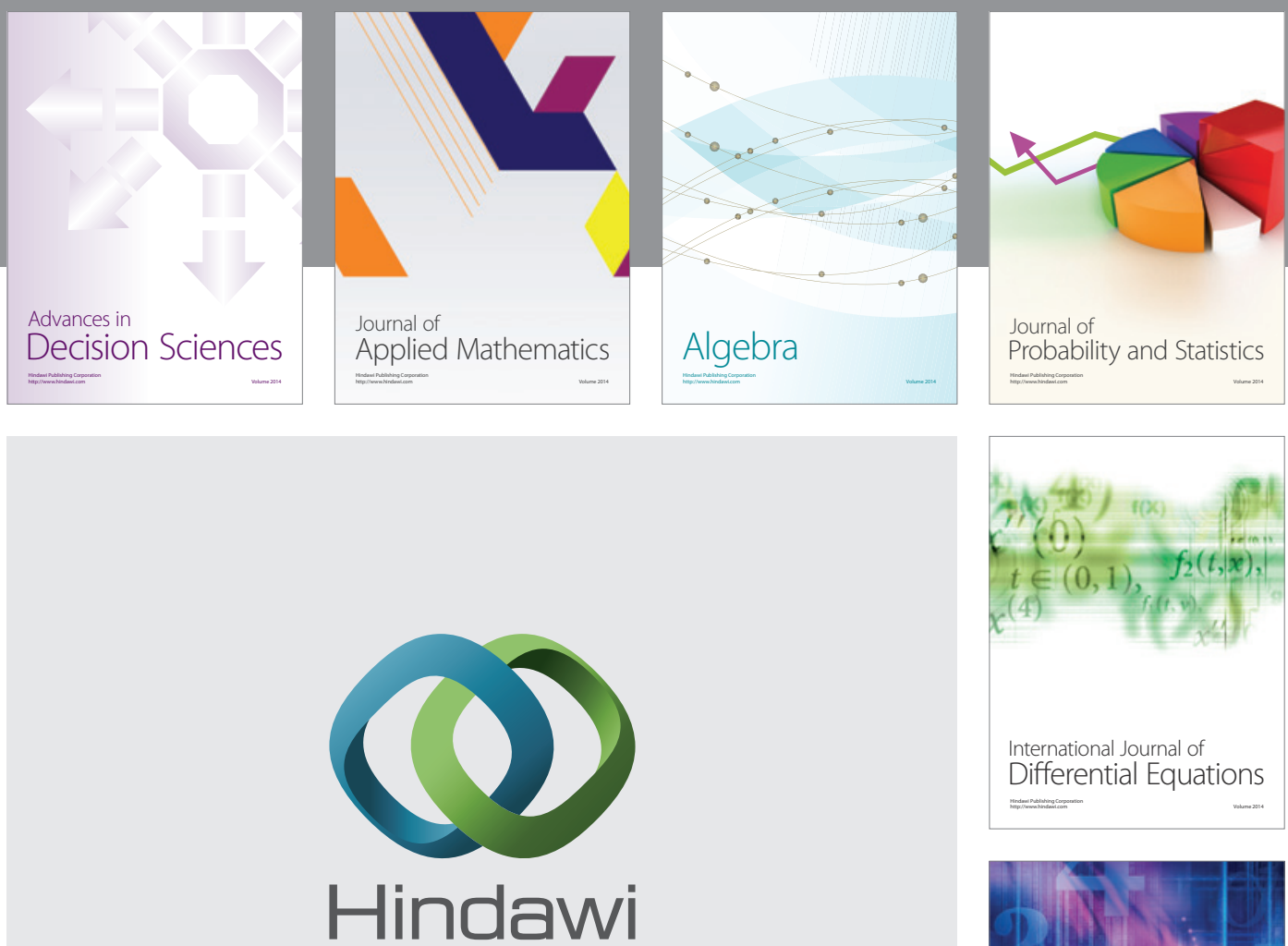

Submit your manuscripts at http://www.hindawi.com
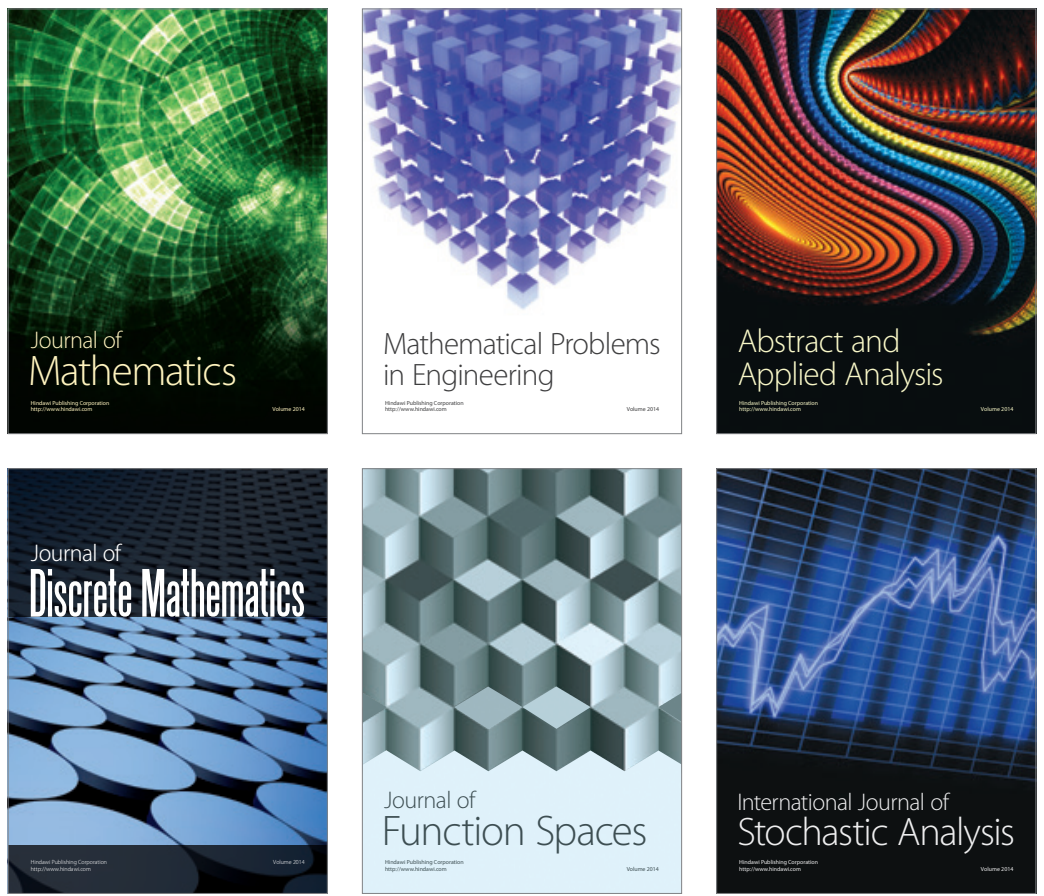

Journal of

Function Spaces

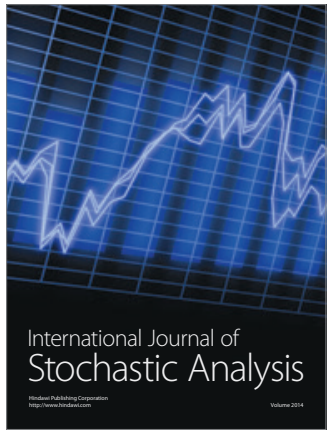

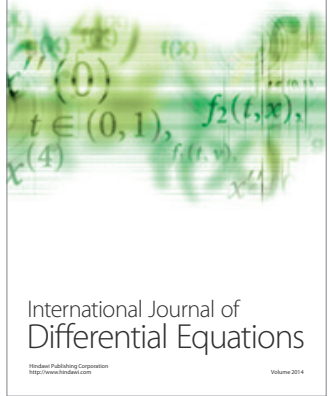
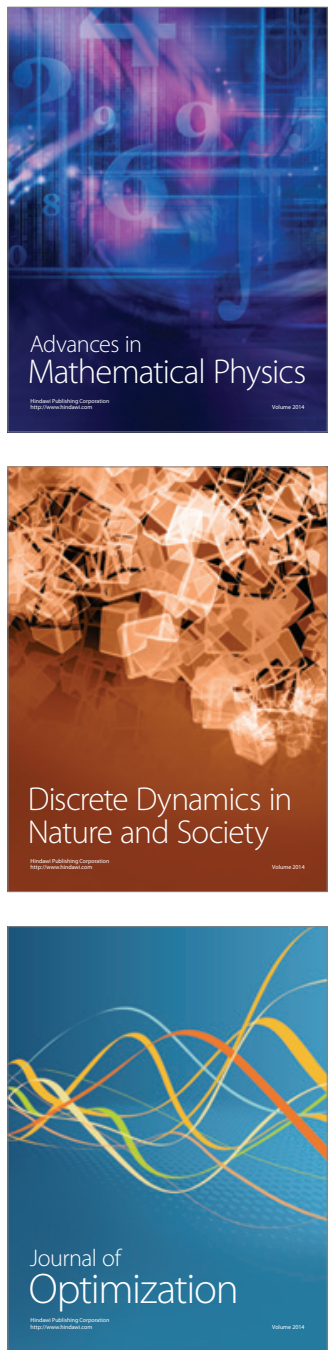